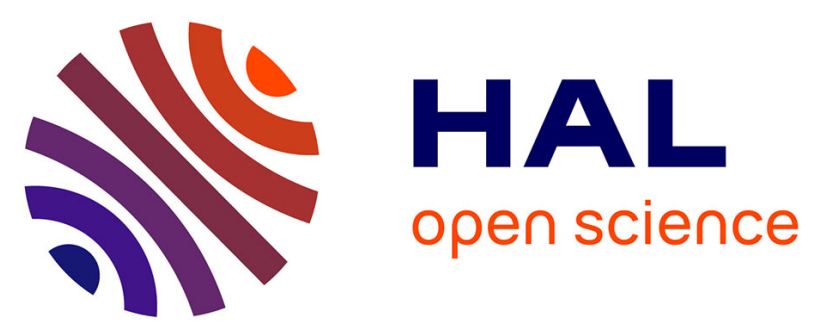

\title{
Quantitative Ultrasound in Ex Vivo Fibrotic Rabbit Livers
}

Emilie Franceschini, Jean-Michel Escoffre, Anthony Novell, Laurent Auboire, Vanda Mendes, Yanis Bénane, Ayache Bouakaz, Olivier Basset

\section{- To cite this version:}

Emilie Franceschini, Jean-Michel Escoffre, Anthony Novell, Laurent Auboire, Vanda Mendes, et al.. Quantitative Ultrasound in Ex Vivo Fibrotic Rabbit Livers. Ultrasound in Medicine \& Biology, 2019, 45 (7), pp.1777-1786. 10.1016/j.ultrasmedbio.2019.02.013 . hal-02200101

\section{HAL Id: hal-02200101 \\ https://hal.science/hal-02200101}

Submitted on 31 Jul 2019

HAL is a multi-disciplinary open access archive for the deposit and dissemination of scientific research documents, whether they are published or not. The documents may come from teaching and research institutions in France or abroad, or from public or private research centers.
L'archive ouverte pluridisciplinaire $\mathbf{H A L}$, est destinée au dépôt et à la diffusion de documents scientifiques de niveau recherche, publiés ou non, émanant des établissements d'enseignement et de recherche français ou étrangers, des laboratoires publics ou privés. 


\title{
Quantitative ultrasound in ex vivo fibrotic rabbit livers
}

\author{
Emilie Franceschini ${ }^{\mathrm{a}, *}$, Jean-Michel Escoffre ${ }^{\mathrm{b}}$, Anthony Novell ${ }^{\mathrm{b}}$, Laurent \\ Auboire $^{\mathrm{b}}$, Vanda Mendes ${ }^{\mathrm{b}}$, Yanis M. Benane ${ }^{\mathrm{c}}$, Ayache Bouakaz ${ }^{\mathrm{b}}$, Olivier \\ Basset $^{\mathrm{c}}$ \\ ${ }^{a}$ Aix-Marseille Université, CNRS, Centrale Marseille, LMA, Marseille, France \\ ${ }^{b}$ UMR 1253, iBrain, Université de Tours, Inserm, Tours, France \\ ${ }^{c}$ Univ Lyon, INSA-Lyon, Université Claude Bernard Lyon 1, UJM-Saint Etienne, \\ CNRS, Inserm, CREATIS UMR 5220, U1206, F-69621, Lyon, France
}

\begin{abstract}
Liver fibrosis is the common result of chronic liver disease. Diagnosis and grading liver fibrosis for patient management is mainly based on blood tests and hepatic puncture-biopsy, which is particularly invasive. Quantitative UltraSound (QUS) techniques provide insight into tissue microstructure and are based on the frequency-based analysis of the signals from biological tissues. This study aims to quantify how spectral-based QUS parameters change with fibrosis grade. The changes in QUS parameters of healthy and fibrotic rabbit liver samples were investigated and were compared to the changes in liver stiffness using shear wave elastography. Overall, the acoustic concentration (EAC) was found to decrease with increasing fibrosis grade while the effective scatterer size (ESD) was found to be higher in fibrotic livers when compared to normal liver. The result of this study indicates that the combination of three QUS parameters (stiffness, ESD and EAC) provides the best classifi-
\end{abstract}

*Corresponding Author: Dr. Franceschini Emilie, Aix-Marseille Université, CNRS, Centrale Marseille, LMA, Marseille, France ; Email, franceschini@lma.cnrs-mrs.fr; Tel: $+33(0) 484524286$ 
cation performance, especially for classifying healthy and fibrotic livers.

Keywords: Quantitative ultrasound, Backscatter coefficient, Stiffness,

Fibrotic liver 


\section{INTRODUCTION}

2 Liver fibrosis results from the chronic damage to the liver tissue in associa3 tion with the excessive accumulation of extracellular matrix (ECM) proteins, 4 which is a hallmark feature of a wide variety of chronic liver diseases (Bataller

5 \& Brenner 2005). In the Western lifestyle countries, the most common causes 6 of hepatic fibrosis are chronic Hepatitis C Virus infection, nonalcoholic steato7 hepatitis and alcohol abuse (Pellicoro et al. 2014). The accumulation of ECM 8 proteins, specifically the collagen, alters the architecture of hepatic tissue by 9 forming fibrous wounds. The subsequent development of nodules of regen-

erating hepatocytes outlines the cirrhosis (Lee et al. 2014). This last one leads to increased intrahepatic resistance to blood flow and hepatocellular dysfunction, which induce a portal hypertension and hepatic insufficiency, respectively.

Hepatic puncture-biopsy is the gold standard method to stage liver fibrosis, a biomarker of advanced liver disease (Lee et al. 2018). However, liver biopsy is an invasive and potentially painful technique. In addition, the heterogeneity of pathological liver tissue may lead to a selection bias of the biopsy (Fernandez-Salazar et al. 2011). In this context, several ultrasound elastography methods (such as transient elastography, acoustic radiation force impulse or shear wave elastography (SWE)) have been developed for the noninvasive assessment of liver stiffness, as a Quantitative UltraSound (QUS) imaging biomarker for detection, staging, and monitoring of liver fibrosis (Tang et al. 2015). Indeed, the collagen deposition and other microstructural changes associated with hepatic fibrosis induce parenchymal rigidity. This liver stiffness increases with higher fibrosis stages. Nowadays, 
the liver stiffness is used to evaluate the gravity of liver disease, to manage treatment decision and to assess response to treatment (Bhat et al. 2017, Chen et al. 2018, Ippolito et al. 2018). Using current histological techniques with hematoxylin/eosin and picrosirius red staining, pathologists classify the different fibrosis stage using ordinal scores (i.e., from stage 0 to stage 6 with Ishak grading) while ultrasound imaging data provide a dichotomized categorization of fibrosis stages including the significant (stage $\geq 2$ ) and advanced (stage $\geq 4$ ) liver fibrosis stages (Tang, et al. 2015). This different classification observed between histological and imaging techniques might be explained by the sensitivity of both techniques. Indeed, pathologists perform a semi-quantitative analysis of the location and amount of excess collagen but also microstructural changes in the liver tissue architecture (Hui et al. 2004, Kleiner et al. 2005, Theise 2007). Such histological analysis demonstrated that there is no linear correlation between the fibrosis stage and the total amount of collagen, thus suggesting that fibrosis stage is not stated exclusively by the total collagen content (Calvaruso et al. 2009, Xu et al. 2014). Indeed, the total amount of collagen remains almost stable from the stage 0 to higher stages, until an advanced fibrosis stage is reached, subsequently the total collagen content exponentially increases. These studies clearly reported that the intra-hepatic location of collagen and the liver tissue remodeling contribute to the fibrosis stage. Consequently, the exponential relationship described between the liver stiffness and the fibrosis stage strongly suggests that ultrasound elastography is more a direct biomarker of total amount of collagen than fibrosis stage.

To overcome these limitations, combinations of non-invasive QUS tech- 
niques can be used to improve accuracy and to provide a comprehensive assessment of different stages of liver fibrosis. Spectral-based QUS techniques using the parameterization of the backscatter coefficient (BSC) have demonstrated success in many preclinical and clinical applications for tissue characterization (Oelze \& Mamou 2016). Specifically, a scattering model is fit to the measured BSC and the fit parameters can provide a meaningful description of the tissue microstructure (i.e., scatterer size, shape, scattering strength and spatial organization). One of the most popular scattering models is the spherical Gaussian model (SGM) developed by Lizzi (Lizzi et al. 1986). This model describes tissue as a random medium composed of spherical structures having continuous spherical impedance fluctuation following a spherical Gaussian curve, and yields two QUS parameters: the average effective scatterer size (ESD) and the acoustic concentration (EAC) (i.e., the product of the scatterer number density and the square of the relative impedance difference between scatterers and the surrounding medium). Some spectral-based QUS studies have been performed to characterize liver fibrosis. In clinical trials, the BSCs at the center frequency of $3 \mathrm{MHz}$ were found to be higher in patients with liver cirrhosis, when compared to a healthy patient group (O’Donnell \& Reilly 1985, Lu et al. 1999). In ex vivo experiments, the attenuation coefficient, sound speed and integrated backscatter coefficient with a $20 \mathrm{MHz}$ pulse-echo system allowed a reasonable segregation of patient groups suffering from different fibrosis grades (Meziri et al. 2005). This study aims to quantify how spectral-based QUS parameters change with fibrosis grade. Liver fibrosis was induced by repeated subcutaneous injections of carbon tetrachloride $(\mathrm{CCl} 4)$ in the neck of rabbits. Attenuation 
and backscatter coefficients were estimated over a wide frequency range (up to $40 \mathrm{MHz}$ ) from fresh rabbit liver samples using the spectral difference method and the reference phantom method, respectively. The two QUS parameters (ESD and EAC) were estimated by fitting the measured BSC to an estimated BSC calculated with the SGM theoretical model. The changes in QUS parameters of healthy and fibrotic livers were investigated and were compared to the changes in liver stiffness using SWE. Finally, we evaluated whether correctly classifying fibrotic livers was possible based on these QUS estimates.

\section{MATERIAL AND METHODS}

Rabbit liver fibrosis model

All procedures were performed in accordance with French and International ethical guidelines and were approved by the National Committee for Animal Care and Ethics in Animal Experiments (No. 20160201160113533872). Eighteen New Zealand white male rabbits (Charles Rivers, Bois des Oncins, France) were housed in a temperature-controlled room $\left(23^{\circ} \mathrm{C}\right)$ with 12:12-h light-dark cycle in isolation cages. Food and water were presents ad libitum.

During the injection protocol and B-mode imaging, the rabbits were anesthetized by $3 \%$ isoflurane (Isoflo, Coveto, Limoges, France) and oxygen via a facial oxygen mask in place throughout the procedure. This anesthesia was limited to $20 \mathrm{~min}$, and a thermostatically controlled pad was used to maintain body temperature at about $37^{\circ} \mathrm{C}$ plate. The rabbits were 9 weeks old at the beginning of the experiment, weighing in average $2.5 \pm 0.2 \mathrm{~kg}$. To 
induce liver fibrosis, a dose of $0.2 \mathrm{~mL} / \mathrm{kg}$ CCl4 (Sigma-Aldrich, St. Louis, MO) as a $50 \%(\mathrm{v} / \mathrm{v})$ solution in olive oil was subcutaneously administered twice a week during several successive weeks (from 3 up to 12 weeks) because the grade of liver fibrosis is related to the duration of $\mathrm{CCl} 4$ injection ( $\mathrm{Li}$ et al. 2018). Experimentation used 18 animals divided in 5 groups. The first 4 groups corresponded to injection protocols of 3 weeks ( $n=4$ rabbits), 6 weeks ( $n=4$ rabbits), 9 weeks ( $n=3$ rabbits) and 12 weeks ( $n=3$ rabbits), and the fifth group ( $n=4$ rabbits) were left untreated to generate control data. The progression of liver fibrosis was monitored twice a week using an Acuson Sequoia ultrasound system (Siemens Healthcare GmbH, Erlangen, Germany) with an Acuson 15L8 ultrasound probe. An expert medical doctor (L.A.) reviewed the ultrasound B-scans of healthy and fibrotic livers.

After liver fibrosis induction, the rabbits were euthanized under anesthesia by i.v. injection of $1 \mathrm{~mL} / \mathrm{kg}$ Doléthal (Vétoquinol S.A., Magny-Vernois, France). For each rabbit, the liver was excised and immersed in degassed saline solution to be ultrasonically imaged for QUS analysis as described below. (Ex vivo study was conducted to allow high frequency measurements up to $40 \mathrm{MHz}$ and to explore liver microstructure properties, as discussed later in the section "Results and Discussion".)

Shear wave elastography (SWE)

Real-time SWE was performed using an Aixplorer UltraFastTM system (SuperSonic Imagine, Aix-en-Provence, France), equipped with a linear array probe (SL15-4) with a center frequency of $8.5 \mathrm{MHz}$ in order to determine the liver stiffness ex vivo. Elasticity estimates were color-coded creating a 2D quantitative SWE image of liver stiffness, which was shown in box form over 
a conventional B-mode image. For each animal, liver stiffness measurements were acquired on all three liver lobes. For each lobe, three round regions of interest (ROIs) were placed in the box on the gray-scale ultrasound image. Liver stiffness was expressed as the mean \pm standard deviation of elastic modulus $(\mathrm{kPa})$ of three liver lobes.

\section{Spectral-based quantitative ultrasound method}

High-frequency ultrasound imaging was performed using the Vevo 2100 imaging system (FUJIFILM - VisualSonics Inc, Toronto, Canada). Two linear array probes, MS250 of center frequency $20 \mathrm{MHz}$ and MS550 of center frequency $40 \mathrm{MHz}$, were used in B-mode in order to estimate tissue microstructures on the three liver lobes of each rabbit. For each liver lobe, ten consecutive frames were acquired on three different locations. One frame is formed from 512 scan lines. The orientation and the position of the probe were chosen to prevent the presence of specular echoes originating from the veins or arteries in the scan plane.

For the two linear array probes, reference scans were acquired from a well-characterized reference phantom after conducting the experiments on rabbit livers with the same imaging system settings (Yao et al. 1990). The reference phantom was provided by the University of Wisconsin (WI, USA) and consisted of $6 \mathrm{~g} / \mathrm{L}$ of glass beads with radii ranging from 0.4 to $6 \mu \mathrm{m}$ in a gel-surrounding medium. The reference phantom has been characterized to measure its backscatter coefficient $\mathrm{BSC}_{\text {ref }}$ on the $10-40 \mathrm{MHz}$ frequency bandwidth using a planar reflector and focused transducers having different center frequencies 10, 20 and $35 \mathrm{MHz}$ (Chen et al. 1997). The measured $\mathrm{BSC}_{\mathrm{ref}}$ magnitude was comparable with backscatter coefficient magnitudes 
lected ROIs placed on the ultrasound B-mode images of liver lobe. The ROIs are drawn as large as possible with at least 200 scan lines. QUS images based on BSC analysis depict tissue properties in a system-independent manner. The reference phantom technique was employed for local attenuation and BSC estimation to account for the electromechanical system response and the depth-dependent diffraction and focusing effects of the ultrasound beam (Labyed \& Bigelow 2011, Yao et al. 1990). The local attenuation $\alpha_{\mathrm{s}}$ was first estimated using the spectral log difference method with the $40-\mathrm{MHz}$ probe (Labyed \& Bigelow 2011). The measured $\mathrm{BSC}_{\text {meas }}$ was then computed using the reference phantom technique with both $20-\mathrm{MHz}$ and $40-\mathrm{MHz}$ probes (Yao et al. 1990). The $\mathrm{BSC}_{\text {meas }}$ of the liver sample was computed as follows:

$$
\mathrm{BSC}_{\text {meas }}(f)=\mathrm{BSC}_{\text {ref }}(f) \frac{\overline{P_{\text {meas }}}(f)}{\overline{P_{\text {ref }}}(f)} e^{4 z\left(\alpha_{\mathrm{s}}(f)-\alpha_{\text {ref }}(f)\right)}
$$

where $f$ is the frequency, $\mathrm{BSC}_{\text {ref }}$ is the $\mathrm{BSC}$ of the reference phantom measured by using a planar reflector and focused transducers (as described previously); $\overline{P_{\text {meas }}}$ and $\overline{P_{\text {ref }}}$ are the power spectra for the liver sample and reference phantom at equivalent depth $z$. The last term (i.e., the exponential function) compensates for attenuation effects. This procedure yielded a $\mathrm{BSC}_{\text {meas }}$ for 
each probe: in the 10-30 MHz bandwidth with the MS250 probe and in the 20-40 MHz bandwidth with the MS550 probe. The resultant two $\mathrm{BSC}_{\text {meas }}$ could be combined to yield a single $\mathrm{BSC}_{\text {meas }}$ over the combined bandwidth of the two transducers (i.e., $10-40 \mathrm{MHz}$ ). More specifically, there is at least one intersection point (at frequency $f_{I}$ ) between the two $\mathrm{BSC}_{\text {meas }}$ curves within the 20-30 MHz frequency bandwidth, such that the two $\mathrm{BSC}_{\text {meas }}$ curves are combined by using the $\mathrm{BSC}_{\text {meas }}$ measured with the MS250 probe in the [10 $\left.\mathrm{MHz}-f_{I}\right]$ bandwidth and the $\mathrm{BSC}_{\text {meas }}$ measured with the MS550 probe in the $\left[f_{I^{-}}-40 \mathrm{MHz}\right]$ bandwidth.

For each ROI, two QUS parameters, namely the ESD and EAC, were obtained by fitting the measured $\mathrm{BSC}_{\text {meas }}$ with the spherical Gaussian model (Insana et al. 1990). The spherical Gaussian model is based on several approximations (Born, far-field, incident plane wave, isotropic medium) for soft tissue scattering. Based on these approximations, the BSC is modeled using a spatial autocorrelation function describing the size, shape, acoustic properties and distribution of the scatterers in the medium; and is expressed as follows (Insana et al. 1990):

$$
\operatorname{BSC}_{\mathrm{SGM}}(k)=\frac{k^{4} V_{s}^{2} n_{z}}{4 \pi^{2}} e^{-2 k^{2} d^{2}}=\frac{k^{4} V_{s}^{2} n_{z}}{4 \pi^{2}} e^{-0.827 k^{2} a^{2}},
$$

where $k$ is the wavenumber, $n_{z}$ is the acoustic concentration (i.e., $\mathrm{EAC}=n_{z}$ ), $d$ is the correlation distance that characterized the continuous isotropic medium and $V_{s}=\left(2 \pi d^{2}\right)^{3 / 2}$. The effective scatterer radius $a$ is related to the correlation distance $d$ by setting values of $V_{s}$ for a continuum model equal to the volume of an effective sphere of radius $a: V_{s}=\left(2 \pi d^{2}\right)^{3 / 2}=(4 / 3) \pi a^{3}$ (Insana et al. 1990). The ESD has been related to the size of dominant scatterers in liver tissues (i.e., $\mathrm{ESD}=2 a$ ). The reported $\mathrm{ESD}$ and $\mathrm{EAC}$ parameters are 
averaged over the three measurements performed on each lobe.

\section{Histopathology}

Livers were fixed in the $4 \%$ formol solution (Labo-moderne, Paris, France). Then, histological samples were embedded in paraffin, cut at approximately $5 \mu \mathrm{m}$, and prepared using conventional hematoxylin/eosin and picrosirius red protocols (Novaxia, Saint-Laurent Nouan, France). These tissue sections were examined by light microscopy on a Leica Diaplan microscope. Histological slides were reviewed by an expert hepatopathologist (Le Net Pathologist Consulting, Amboise, France). The liver fibrosis was graded according to the Ishak grading (Ishak et al. 1995), a grading with a 7-tier scale offering high discriminant descriptive power suitable for research purposes (Almpanis et al. 2016).

\section{RESULTS AND DISCUSSION}

Effects of $\mathrm{CCl}_{4}$ on rabbit behavior and on liver anatomy

Upon visual inspection of the rabbits, all CCl4 treated animals were generally less groomed and less active compared to untreated control group. In addition, 3 CCl4 treated animals were dead before the study ended and a significant loss of body weight (i.e., $>10 \%$ of initial body weight) was also observed in 7 out of 15 surviving animals in the first three weeks of CCl4 injection (Table 1).

In the present study, B-mode ultrasound imaging was chosen to monitor the effects of CCl4 administration on liver anatomy. During the two first weeks of CCl4 injection, neither liver failure nor behavioral anomalies (i.e., 
loss of appetite, dehydration, loss of body weight, breathing difficulties) was observed in all studied rabbits. However, after 3 weeks of CCl4 injection, abdominal ultrasound imaging revealed an increase in the liver echogenicity in all CCl4 treated animals. Figures 1(a) and 1(b) show representative Bmode images of healthy and fibrotic (grade 5) liver with 12-weeks of CCl4 injection. The fibrotic liver shown in Fig. 1(b) presents an accumulation of fluids in the abdominal cavity, also called ascites, that suggests a hepatic failure. The qualitative analysis of abdominal images from all the studied rabbits should suggest that the amount of fluid in the abdominal cavity increased with the number of $\mathrm{CCl} 4$ injections. At the study end, the necropsy confirmed the presence of ascites in the abdominal cavity of all $\mathrm{CCl} 4$ injected animals. In addition, these rabbits had a strong discoloration of the liver tissues in comparison with the control group, as it can be observed in Figs. $1(\mathrm{c})$ and $1(\mathrm{~d})$.

\section{Histopathological analysis}

To confirm the induction of liver fibrosis, histopathological analyzis was performed on liver tissues. As shown in Figure 2, the picrosirius red staining clearly indicates an excessive accumulation of collagen around the lobule structures confirming that the repetitive administrations of $\mathrm{CCl} 4$ induced liver fibrosis. Despite intra- and inter-individual variations in the liver response to $\mathrm{CCl} 4$ administration were observed, the amount of fibrosis was homogeneous among three liver lobes for each rabbit. In addition, minimal haemosiderosis (i.e., indicator of previous hemorrhage) was present in all treated animals. Table 2 gives the relationship between the fibrosis grades and the weeks of $\mathrm{CCl} 4$ injection. The amount of fibrosis increases with the 
duration of CCl4 injection protocol, as expected. Based on Ishak grading, the rabbit livers were classified within different grades (denoted $G$ in the following): G0 with no fibrosis (4 rabbits), G1 (2 rabbits), G2 (2 rabbits), G3 (2 rabbits), G4 (3 rabbits) and G5 (2 rabbits). No liver cirrhosis (G6) was detected.

\section{QUS parameter estimates}

Typical examples of shear wave elastography images are shown in Fig. 3, and the average Young's modulus of all grades are summarized in Table 3. Young's modulus values were on the order of several thousands of Pascals and noticeably increased with the fibrosis grade, as expected. The statistical analysis using the Pearson correlation method reveals that the liver fibrosis score was positively correlated with the Young's modulus $(p<0.0001)$, as expected.

The attenuation was found to depend linearly on frequency, within the 20-40 MHz frequency bandwidth. The mean values and standard deviation of attenuation coefficients were equal to $0.58 \pm 0.13,0.85 \pm 0.07,0.87 \pm 0.09$, $0.83 \pm 0.08,0.89 \pm 0.07$ and $0.91 \pm 0.09 \mathrm{~dB} / \mathrm{cm} / \mathrm{MHz}$ for $\mathrm{G} 0$ (normal), G1, G2, G3, G4 and G5, respectively. Averaged attenuation coefficient was thus found to be higher in fibrotic livers. This trend correlates well with previous experimental results conducted at $20 \mathrm{MHz}$ by (Meziri et al. 2005): attenuation coefficients were found to be equal to $0.76 \mathrm{~dB} / \mathrm{cm} / \mathrm{MHz}$ and 1.06 $\mathrm{dB} / \mathrm{cm} / \mathrm{MHz}$ for normal and cirrhotic livers, respectively.

Typical examples of measured $\mathrm{BSC}_{\text {meas }}$ are shown in Fig. 4. The $\mathrm{BSC}_{\text {meas }}$ magnitudes from fibrotic livers were greater than those from normal liver in the 10-22 MHz frequency bandwidth, as observed previously in the literature 
(O'Donnell \& Reilly 1985, Lu et al. 1999). Table 3 also displays the average and standard deviations of the spectral-QUS estimates for the fibrotic grade livers. Overall, the EAC decreased with increasing fibrosis grade and the ESD was found to be higher in fibrotic livers when compared to normal liver for both $10-20 \mathrm{MHz}$ and 10-40 MHz frequency bandwidths.

The abilities of QUS parameters to differentiate among grades of fibrosis were assessed using 1-way ANOVA. Statistically significant differences were quantified using $p$ values $<0.05$. The $p$ values are shown in Table 3. For example, $p$-values for G0 versus (vs) G1 refers to the significance of QUS parameters estimated from animals with no fibrosis with G0 and fibrosis with G1; and $p$-values for [G1-2] vs [G3-5] refers to the significance of QUS parameters estimated from animals with moderate fibrosis with G1 and G2 (denoted G1-2) and with severe fibrosis $\geq$ G3 (denoted G3-5). The Young's modulus, ESD and EAC parameters ( $p$ value $<0.05)$ can be used to differentiate between G0 and G1, and to differentiate between normal and fibrotic livers. The Young's modulus and EAC parameters $(p$ value $<0.05)$ can be used to differentiate between moderate (G1-2) and severe (G3-5) fibrosis (but not the ESD). None of the parameters enables the graduation of the fibrosis in 6 grades (from G0 to G5). Only the ESD and EAC parameters estimated at high frequencies using the 10-40 MHz could differentiate G2 and G3.

\section{Usefulness of high frequency measurements}

To our knowledge, it is the first time that high frequency measurements up to $40-\mathrm{MHz}$ center frequency probe are reported in ex vivo livers. High frequency measurements cannot be used in real clinical condition, but are useful to obtain a more robust estimation of the scatterer size and to progress fur- 
ther in the understanding of backscattering from fibrotic livers. It is very interesting to observe the frequency-dependent of the $\mathrm{BSC}_{\text {meas }}$ in the case of normal liver that shows a peak of magnitude around $30 \mathrm{MHz}$ (see black curve in Fig. 4). The ESD estimated with the SGM was found to be equal to 19.5 $\mu \mathrm{m}$ in the $10-40 \mathrm{MHz}$ frequency bandwidth. However, it is known that the SGM may not bring a meaningful description of the tissue microstructure in the case of complex media, such as dense and/or polydisperse scattering media (Franceschini et al. 2016). In order to better interpret this peak around $30 \mathrm{MHz}$, the $\mathrm{BSC}_{\text {meas }}$ was fitted with the polydisperse structure factor model, allowing the simultaneous estimation of the scatterer diameter distribution, the volume fraction and the relative impedance contrast (Franceschini et al. 2016). As shown in Fig. 4, the polydisperse structure factor model suggests that this peak is representative of $\mathrm{ESD} \approx 28 \mu \mathrm{m}$, which is quite close from the hepatocyte diameter $d \approx 26 \mu \mathrm{m}$ (as deduced from the area surface of hepatocytes observed in histological slices). However, even with high frequency measurements and advanced scattering models (such as the structure factor model), we were not able to establish a relationship between QUS scatterer property estimates and actual fibrotic liver structures. Note that the QUS parameters obtained from the structure factor model with fibrotic livers were not shown in the present study because the SGM was found to be more efficient than the structure factor model for grading the fibrotic liver (data not shown).

Figure 5 shows typical examples of B-mode images obtained from ex vivo healthy and fibrotic livers using the $40-\mathrm{MHz}$ center frequency probe. The Bmode images of healthy liver display hyper-echogenic structures with hexag- 
onal shapes corresponding to liver lobule structures, whereas fibrotic livers show more homogeneous speckle. So the connective tissue of lobular boundaries plays also an important contribution in the scattering process, in addition to the contribution of the cellular/hepatocyte scattering. Future studies should be focused on the understanding of scattering contribution from connective tissue of lobular boundaries, collagen fibers and cellular/hepatocyte microstructure in normal and fibrotic livers, using for example acoustic models of tissue microstructure, termed impedance maps (Mamou et al. 2005, Luchies \& Oelze 2005, Tamura et al. 2017)

Fibrosis classification based on QUS estimates

The ability of Young's modulus and spectral-QUS parameters for classifying liver grades was studied using the Gaussian Mixture Model (GMM) (McLachlan \& Peel 2000). For this study, we use only the ESD and EAC estimated within the 10-20 MHz bandwidth, which corresponds to a more accessible frequency bandwidth in usual ultrasound devices. The classification was performed by fitting a Gaussian mixture distribution model to the $N$ classes of the QUS data. The classification was tested for three classes $(N=3)$ : no fibrosis with G0 (4 animals), moderate fibrosis with G1 and G2 (4 animals) and severe fibrosis with G3, G4 and G5 (7 animals). When considering the stiffness parameter or the tissue microstructure parameters (ESD and $\mathrm{EAC}$ ), the classification was first performed by using all the 135 data (corresponding to 15 animals $\times 3$ lobes/animal $\times 3$ measurements/lobe). The data were separated in two groups: half of the 135 data were assigned randomly to a training group, and the other half was assigned to a validation group to test the classification on the model obtained with the training group. Figures (6a- 
b) represent the confusion matrices that summarize the classification results when considering the Young's modulus alone and the spectral-QUS parameters alone. When combining the three QUS parameters (Young's modulus, ESD and EAC), the classification was performed by using the QUS parameters averaged over the three measurements for each studied lobe (i.e., 45 measurements corresponding to 15 animals $\times 3$ lobes/animals), because the stiffness and tissue microstructure measurements were not performed on the same ROI. In that case, the separation in training and validation groups was not performed because of the low number of data but a classification using the leave-one-out cross-validation was used. For comparison purpose, the GMM associated with the leave-one-out cross-validation was used by processing the same 45 averaged measurements when considering the stiffness parameter alone and the spectral-QUS parameters alone. The confusion matrices obtained from leave-one-out cross-validation are given in Figs. (6c-e), when using the Young's modulus alone, the spectral-QUS parameters alone, and the combination of spectral-QUS parameters with Young's modulus, respectively.

Overall, the separation in training and validation groups allows us to better classifying the lobes when compared to the leave-one-out cross-validation (see Figs. 6a-d). It can be observed that the class G0 is always better classified ( $P_{c} \geq 83 \%$ of correctly classified lobes) when the spectral-QUS parameters (ESD and EAC) are considered, whatever the classification methods used (see Fig. 6b for the separation in training and validation groups, and see Figs. 6(d-e) for the leave-one-out cross-validation). The fibrotic livers (classes G1-2 and G3-5) are also better classified using the combination of 
the three QUS parameters (Young's modulus, ESD and EAC), when compared to the classifications obtained with the spectral-QUS parameters or with the Young's modulus alone (Figs. 6(c-e)).

The results obtained in the present study suggest that the combination of spectral-QUS parameters with Young's modulus resulted in improved classification compared to Young's modulus alone. Further study should be conducted on in vivo livers to confirm the added value of the spectra-QUS parameters for fibrosis classification. Indeed, the present study is limited to ex vivo liver samples, such that the BSC data were simply compensated for liver attenuation. Challenges for in vivo application are to correctly account for attenuation effects and transmission losses. Even if previous studies have demonstrated the ability to accurately estimate the BSC and attenuation using the reference phantoms on clinical systems (Wirtzfeld et al. 2010, Han et al. 2017), the QUS parameter variance from in vivo application may be higher when compared to those obtained in the present ex vivo study and this could affect the classification efficiency.

\section{Conclusion}

In summary, the EAC was found to decrease with increasing fibrosis grade and the ESD was found to be higher in fibrotic livers when compared to normal liver. The EAC parameter was shown to be more sensitive to changes in fibrotic tissues when compared to ESD parameter, especially for differentiating between moderate (G1-2) and severe (G3-5) fibrosis. The results reported here also suggest that ESD and EAC bring complementary information to standard stiffness measurements for fibrosis classification, since 
375 the combination of the three QUS parameters (Young's modulus, ESD and 376 EAC) provides the best classification performance. Further study should be 377 performed to demonstrate the superiority of the QUS approach combining 378 tissue microstructure and stiffness in human clinical study for classifying liver 379 fibrosis.

\section{Acknowledgements}

The authors acknowledge Mrs V. Schubnel (PST Animalerie, Universit de Tours, France) for the animal experience. We are grateful for the technical support from Visualsonics. This work was supported by the French National Research Agency under grant ANR TecSan 11-008-01 (OB), and ANR-11IDEX-0001-02 (EF) and Inserm grant (JME, AB). 
Almpanis Z, Demonakou M, Tiniakos D. Evaluation of liver fibrosis: "Something old, something new...". Annals of gastroenterology 2016; $29: 445-53$

Bataller R, Brenner DA. Liver fibrosis. J. Clin. Invest. 2005; 115:1100-00

Bhat M, Tazari M, Sebastiani G. Performance of transient elastography and serum fibrosis biomarkers for non-invasive evaluation of recurrent fibrosis after liver transplantation: A meta-analysis. PLoS One 2017; 12:e0185192

Calvaruso V, Burroughs AK, Standish R, Manousou P, Grillo F, Leandro G, Maimone S, Pleguezuelo M, Xirouchakis I, Guerrini GP, Patch D, Yu D, O'Beirne J, Dhillon AP. Computer-assisted image analysis of liver collagen: relationship to Ishak scoring and hepatic venous pressure gradient. Hepatology 2009; 49:1236-44

Chen SH, Lai HC, Chiang IP, Su WP, Lin CH, Kao JT, Chuang PH, Hsu WF, Wang HW, Chen HY, Huang GT, Peng CY. Changes in liver stiffness measurement using acoustic radiation force impulse elastography after antiviral therapy in patients with chronic hepatitis C. PLoS One 2018; 13:e0190455

Chen X, Phillips D, Schwarz KQ, Mottley JG, Parker KJ. The measurement of backscatter coefficient from a broadband pulse-echo system: a new formulation. IEEE Trans Ultras Ferroelectr Freq Control 1997; 44:515-25 Fernandez-Salazar L, Velayos B, Aller R, Lozano F, Garrote JA, Gonzalez JM. Percutaneous liver biopsy: patients' point of view. Scand. J. Gastroenterol. 2011; 46:727-31.

Franceschini E, de Monchy R, Mamou J. Quantitative characterization of 
tissue microstructure in concentrated cell pellet biophantoms based on the structure factor model. IEEE Trans Ultras Ferroelectr Freq Control 2016; 63:1321-1334.

Han A, Andre MP, Erdman JW, Loomba R, Sirlin CB, O'Brien WD. Repeatability and reproducibility of a clinically based QUS phantom study and methodologies. IEEE Trans Ultras Ferroelectr Freq Control 2017; 64:218-231

Hui AY, Liew CT, Go MY, Chim AM, Chan HL, Leung NW, Sung JJ. Quantitative assessment of fibrosis in liver biopsies from patients with chronic hepatitis B. Liver Int 2004; 24:611-8

Insana MF, Wagner RF, Brown DG, Hall TJ. Describing small-scale structure in random media using pulse-echo ultrasound. The Journal of the Acoustical Society of America 1990; 87:179-92

Ippolito D, Schiavone V, Talei Franzesi CR, Casiraghi AS, Drago SG, Riva L, Sironi S. Real-time elastography: Noninvasive diagnostic tool in the evaluation of liver stiffness in patients with chronic viral hepatitis, correlation with histological examination. Dig Dis 2018:1-9

Ishak K, Baptista A, Bianchi L, Callea F, De Groote J, Gudat F, Denk H, Desmet V, Korb G, MacSween RN, Philips J, Portmann BG, Poulsen H, Scheuer PJ, Schmid M, Thaler H. Histological grading and staging of chronic hepatitis. Journal of hepatology 1995; 22:696-9 Kleiner DE, Brunt EM, Van Natta M, Behling C, Contos MJ, Cummings OW, Ferrell LD, Liu YC, Torbenson MS, Unalp-Arida A, Yeh M, McCullough AJ, Sanyal AJ, Nonalcoholic Steatohepatitis Clinical Research N. Design and validation of a histological scoring system for nonalcoholic 
fatty liver disease. Hepatology 2005; 41:1313-21

Labyed Y, Bigelow TA. A theoretical comparison of attenuation measurement techniques from backscattered ultrasound echoes. J Acoust Soc Am $2011 ; 129: 2316-24$

Lee S-J, Kim K-H, Park K-K. Mechanisms of fibrogenesis in liver cirrhosis: The molecular aspects of epithelial-mesenchymal transition. World J Hepatol 2014; 6:207-16

Li RK, Ren XP, Yan FH, Qiang JW, Lin HM, Tao W, Zhao HF, Chen WB. Liver fibrosis detection and staging: a comparative study of T1rho MR imaging and 2D real-time shear-wave elastography. Abdominal radiology 2018; 43:1713-1722

Lizzi FL, Ostromogilsky M, Feleppa EJ, Rorke MC, Yaremko MM. Relationship of ultrasonic spectral parameters to features of tissue microstructure. IEEE Trans Ultrason Ferroelect Freq Contr. 1986; 33:319-329

Lu ZF, Zagzebski JA, Lee FT. Ultrasound backscatter and attenuation in human liver with diffuse disease. Ultrasound Med. Biol. 1999; 25:1047-1054 Luchies A, Oelze ML, Using two-dimensional impedance maps to study weak scattering in sparse random media, J Acoust Soc Am 2016; 139:15571564 .

McLachlan G, Peel D. Finite Mixture Models. Hoboken, NJ: John Wiley \& Sons, Inc., 2000.

Madsen EL, Zagzebski JA, Insana MF, Burke TM, Frank G. Ultrasonically tissue-mimicking liver including the frequency dependence of backscatter. Med Phys 1982; 9:703-10

Mamou J, Oelze ML, O’Brien WD, Zachary JF. Identifying ultrasonic 
scattering sites from three-dimensional impedance maps. J. Acoust. Soc. Am. 2005; 117: 413-423.

Meziri M, Pereira WCA, Abdelwahab A, Degott C, Laugier P. In vitro chronic hepatic disease characterization with a multiparametric ultrasonic approach, Ultrasonics 2005: 43:305-313

Oelze M, Mamou J. Review of quantitative ultrasound: envelope statistics and backscatter coefficient imaging and contributions to diagnostic ultrasound, IEEE Trans Ultrason Ferroelect Freq Contr. 2016; 63:336-351

O'Donnell M, Reilly HF. Clinical evaluation of the B scan. IEEE Trans. Sonics and Ultrasonics 1985; SU-32:450-457

Pellicoro A, Ramachandran P, Iredale JP, Fallowfield JA. Liver fibrosis and repair: immune regulation of wound healing in a solid organ. Nat. Rev. Immunol. 2014; 14:181-94

Tang A, Cloutier G, Szeverenyi NM, Sirlin CB. Ultrasound Elastography and MR Elastography for Assessing Liver Fibrosis: Part 1, Principles and Techniques. AJR Am J Roentgenol 2015; 205:22-32

Tang A, Cloutier G, Szeverenyi NM, Sirlin CB. Ultrasound Elastography and MR Elastography for Assessing Liver Fibrosis: Part 2, Diagnostic Performance, Confounders, and Future Directions. AJR Am J Roentgenol $2015 ; 205: 33-40$

Tamura K, Franceschini E, Mamou J, Yamaguchi T. Structure factor model-based approach for analyzing two-dimensional impedance map and studying scattering from polydisperse dense media, IEEE International Ultrasonics Symposium 2017, 10.1109/ULTSYM.2017.8092125

Theise ND. Liver biopsy assessment in chronic viral hepatitis: a personal, 
486

487

practical approach. Mod Pathol 2007; 20 Suppl 1:S3-14

Wirtzfeld LA, Cross-imaging platform comparison of ultrasonic backscatter coefficient measurements of live rat tumors. J Ultrasound Med 2010; 29:11171123.

Xu S, Wang Y, Tai DCS, Wang S, Cheng CL, Peng Q, Yan J, Chen Y, Sun J, Liang X, Zhu Y, Rajapakse JC, Welsch RE, So PTC, Wee A, Hou J, Yu H. qFibrosis: a fully-quantitative innovative method incorporating histological features to facilitate accurate fibrosis scoring in animal model and chronic hepatitis B patients. Journal of hepatology 2014; 61:260-69 Yao LX, Zagzebski JA, Madsen EL. Backscatter coefficient measurements using a reference phantom to extract depth-dependent instrumentation factors. Ultrasonic imaging 1990; 12:58-70. 


\section{${ }_{498}$ Tables}

499 Table 1: Effect of CCl4 on survival and loss of body weight.

Loss of body weight

\begin{tabular}{cccc} 
& \multicolumn{2}{c}{$(>10 \%$ initial $)$} & \\
\cline { 2 - 3 } Weeks of CCl4 & $\leq 3$ weeks & Study & \\
injection & of CCl4 & end & Survival \\
\hline 0 & $0 / 4$ & $0 / 4$ & $4 / 4$ \\
3 & $1 / 2^{a}$ & $0 / 2^{a}$ & $2 / 4$ \\
6 & $0 / 3^{b}$ & $0 / 3^{b}$ & $3 / 4$ \\
9 & $3 / 3$ & $0 / 3$ & $3 / 3$ \\
12 & $3 / 3$ & $0 / 3$ & $3 / 3$ \\
\hline
\end{tabular}

${ }^{a}$ Two or ${ }^{b}$ one rabbits are dead before the third week of CCl4 injection. 
501 Table 2: Grade of fibrosis in the liver according to the Ishak grading.

502

\begin{tabular}{cccccc}
\hline Weeks of CCl4 injection & 0 & 3 & 6 & 9 & 12 \\
\hline Number of rabbits & 4 & 2 & 3 & 3 & 3 \\
\hline Grade 0 (control) & 4 & - & - & - & - \\
Grade 1 & - & 2 & - & - & - \\
Grade 2 & - & - & 2 & - & - \\
Grade 3 & - & - & 1 & 1 & - \\
Grade 4 & - & - & - & 1 & 2 \\
Grade 5 & - & - & - & 1 & 1 \\
Grade 6 (cirrhosis) & - & - & - & - & - \\
\hline
\end{tabular}




\begin{tabular}{cccccc}
\hline \multirow{2}{*}{$\begin{array}{c}\text { Fibrosis } \\
\text { grade }\end{array}$} & Young's & \multicolumn{2}{c}{ QUS parameters 10-20 MHz } & \multicolumn{2}{c}{ QUS parameters 10-40 MHz } \\
\cline { 3 - 6 } & modulus & ESD & EAC & ESD & EAC \\
Grade 0 & $5.89 \pm 1.34$ & $22.6 \pm 1.9$ & $49.0 \pm 2.3$ & $19.5 \pm 1.2$ & $52.3 \pm 1.3$ \\
Grade 1 & $7.93 \pm 1.62$ & $34.9 \pm 2.4$ & $45.9 \pm 1.5$ & $26.7 \pm 0.7$ & $50.6 \pm 1.0$ \\
Grade 2 & $9.25 \pm 1.76$ & $31.8 \pm 2.4$ & $45.7 \pm 1.8$ & $25.0 \pm 1.2$ & $50.4 \pm 0.5$ \\
Grade 3 & $10.07 \pm 1.83$ & $31.0 \pm 1.6$ & $44.7 \pm 0.8$ & $26.1 \pm 0.9$ & $47.5 \pm 0.9$ \\
Grade 4 & $11.46 \pm 1.94$ & $33.6 \pm 3.0$ & $44.6 \pm 1.1$ & $26.1 \pm 2.0$ & $48.9 \pm 1.0$ \\
Grade 5 & $12.99 \pm 2.07$ & $33.4 \pm 3.1$ & $43.9 \pm 1.3$ & $25.9 \pm 1.3$ & $48.3 \pm 0.7$ \\
\hline$p$-values & & & & & \\
G0 vs G1 & 0.0026 & 0.0001 & 0.0320 & 0.0001 & 0.0135 \\
G0 vs [G1-2] & 0.0000 & 0.0000 & 0.0068 & 0.0000 & 0.0001 \\
G0 vs [G3-5] & 0.0000 & 0.0000 & 0.0000 & 0.0000 & 0.0000 \\
[G1-2] vs [G3-5] & 0.0000 & 0.5926 & 0.0484 & 0.9559 & 0.0000 \\
G2 vs G3 & 0.3939 & 0.6991 & 0.3939 & 0.0931 & 0.0022 \\
\hline
\end{tabular}




\section{Figure Captions}

Figure 1: In vivo and ex vivo observations of healthy and fibrotic livers. Representative anatomical ultrasound images of healthy (a) and fibrotic (grade 5) liver (b). Representative macroscopic images of healthy (c) and fibrotic (grade 5) liver (d).

Figure 2: Examples of histological images of healthy and fibrotic livers stained using hematoxylin/eosin and picrosirius red.

Figure 3: Representative shear wave elastography images of ex vivo healthy and fibrotic livers.

Figure 4: Typical examples of measured BSCs from ex vivo healthy and fibrotic livers using the two probes of center frequencies $20-\mathrm{MHz}$ and 40-MHz. Also represented are the fitted curves with the SGM (dotted line) and with the polydisperse Structure Factor Model SFM (dashed line) for the healthy liver. The SGM estimates the ESD and EAC, whereas the polydisperse SFM estimates the scatterer size distribution, the volume fraction $\phi$ and the relative impedance difference $\gamma_{z}$.

Figure 5: Representative B-mode images obtained from ex vivo healthy and fibrotic livers using the probe of center frequency 40-MHz.

Figure 6: (a) and (b) Confusion matrices obtained from the separation in training and validation groups when considering the Young's modulus $E$ alone and the spectral-QUS parameters alone. (c), (d) and (e) Confusion matrices obtained from the leave-one-out cross-validation when considering the Young's modulus E alone, the spectral-QUS parameters 
531

532

533

alone and the combination of spectral-QUS parameters with Young's modulus. Also given are the percentages of correctly classified lobes, denoted $P_{c}$. 
(a)
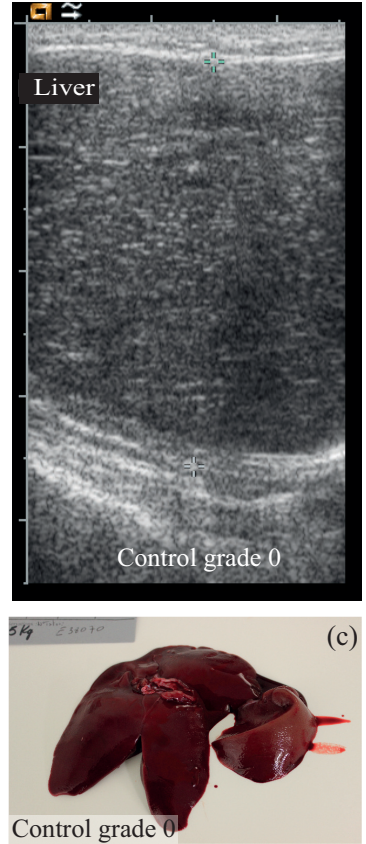

(b)

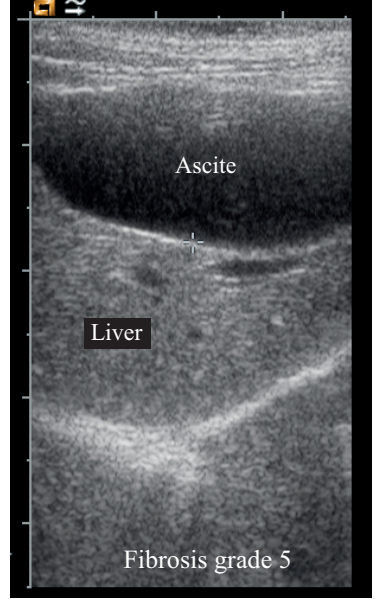

(d)

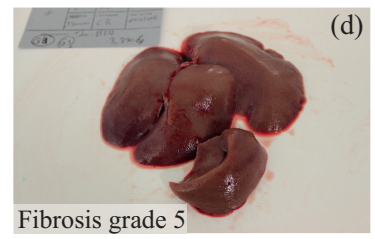

Figure 1: In vivo and ex vivo observations of healthy and fibrotic (grade 5) livers. Representative anatomical ultrasound images of healthy (a) and fibrotic liver (b). Representative macroscopic images of healthy (c) and fibrotic liver (d). 

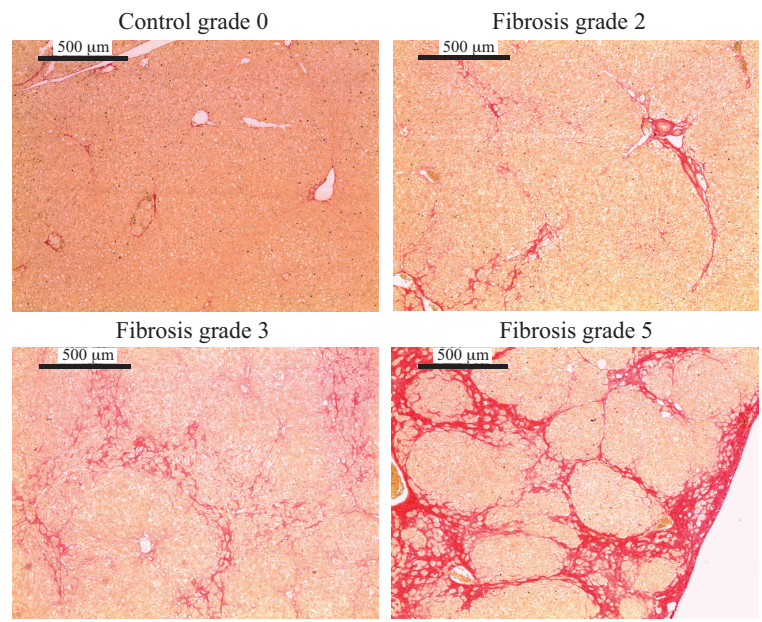

Figure 2: Examples of histological images of healthy and fibrotic livers stained using hematoxylin/eosin and picrosirius red. 


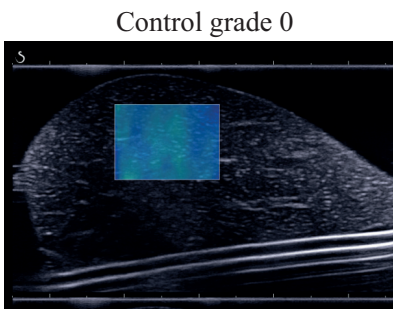

Fibrosis grade 2

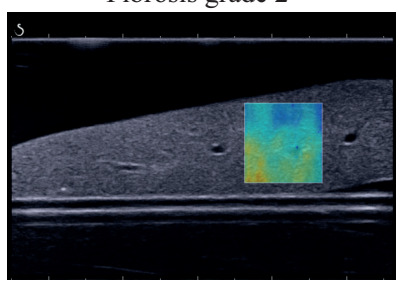

Fibrosis grade 5

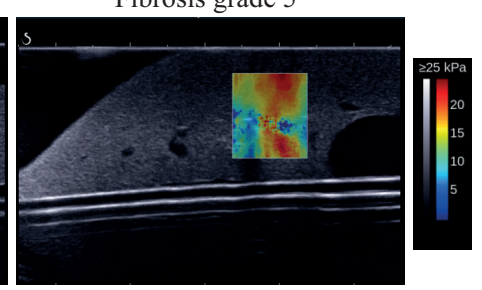

Figure 3: Representative shear wave elastography images of ex vivo healthy and fibrotic livers. 


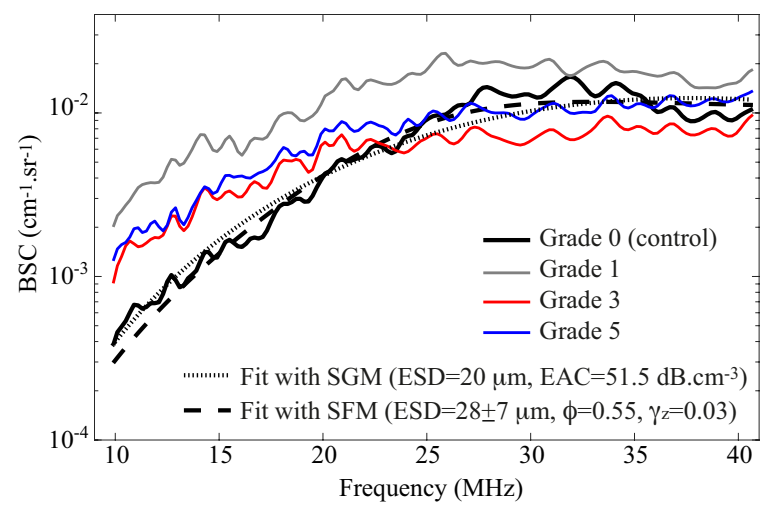

Figure 4: Typical examples of measured BSCs from ex vivo healthy and fibrotic livers using the two probes of center frequencies $20-\mathrm{MHz}$ and $40-\mathrm{MHz}$. Also represented are the fitted curves with the SGM (dotted line) and with the polydisperse Structure Factor Model SFM (dashed line) for the healthy liver. The SGM estimates the ESD and EAC, whereas the polydisperse SFM estimates the scatterer size distribution, the volume fraction $\phi$ and the relative impedance difference $\gamma_{z}$. 


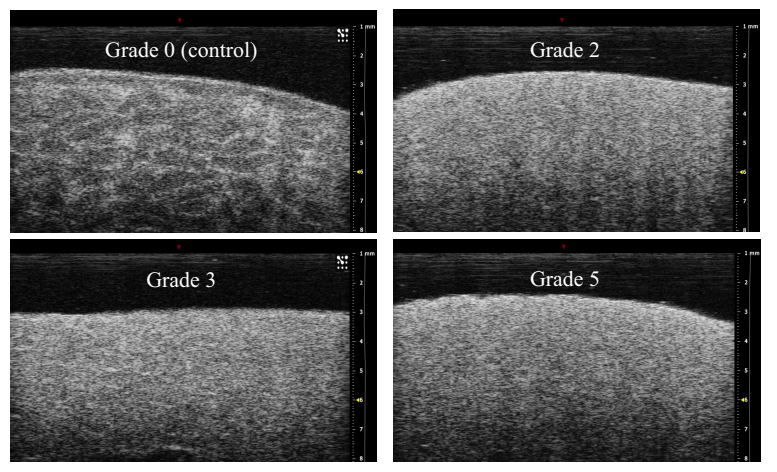

Figure 5: Representative B-mode images obtained from ex vivo healthy and fibrotic livers using the probe of center frequency 40-MHz. 
a) Young's modulus $E$

\begin{tabular}{|c|c|c|c|c|c|}
\hline & \multicolumn{3}{|c|}{ Assigned } & \multirow{2}{*}{$P_{c}$} \\
\hline & & G0 & G1-2 & G3-5 & \\
\hline \multirow{3}{*}{ 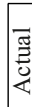 } & G0 & 14 & 4 & 0 & $77.8 \%$ \\
\hline & G1-2 & 0 & 15 & 3 & $83.3 \%$ \\
\hline & G3-5 & 0 & 4 & 27 & $87.1 \%$ \\
\hline
\end{tabular}

c) Young's modulus $E$

\begin{tabular}{|c|c|c|c|c|c|}
\hline & \multicolumn{3}{|c|}{ Assigned } & \multirow{2}{*}{$P_{c}$} \\
\hline & & G0 & G1-2 & G3-5 & \\
\hline \multirow{3}{*}{ 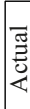 } & G0 & 8 & 4 & 0 & $66.7 \%$ \\
\hline & G1-2 & 0 & 11 & 1 & $91.7 \%$ \\
\hline & $\overline{\text { G3-5 }}$ & 0 & 7 & 14 & $66.7 \%$ \\
\hline
\end{tabular}

b) ESD and EAC (10-20 MHz)

\begin{tabular}{|c|c|c|c|c|c|}
\hline & \multicolumn{3}{|c|}{ Assigned } & \multirow{2}{*}{$P_{c}$} \\
\hline & & G0 & G1-2 & G3-5 & \\
\hline \multirow{3}{*}{$\begin{array}{l}{ }_{0}^{\pi} \\
\\
\end{array}$} & G0 & 18 & 0 & 0 & $100 \%$ \\
\hline & G1-2 & 0 & 12 & 6 & $66.7 \%$ \\
\hline & G3-5 & 0 & 7 & 24 & $77.4 \%$ \\
\hline
\end{tabular}

d) ESD and EAC (10-20 MHz)

\begin{tabular}{|c|c|c|c|c|c|}
\hline & \multicolumn{3}{|c|}{ Assigned } & \multirow{2}{*}{$P_{c}$} \\
\hline & & G0 & G1-2 & G3-5 & \\
\hline \multirow{3}{*}{$\begin{array}{l}\stackrel{\pi}{己} \\
\stackrel{0}{0} \\
\end{array}$} & G0 & 10 & 0 & 2 & $83.3 \%$ \\
\hline & G1-2 & 0 & 7 & 5 & $58.3 \%$ \\
\hline & G3-5 & 0 & 8 & 13 & $61.9 \%$ \\
\hline
\end{tabular}

e) Young's modulus $E$,

ESD and EAC (10-20 MHz)

\begin{tabular}{|c|c|c|c|c|c|}
\hline & \multicolumn{3}{|c|}{ Assigned } & \multirow{2}{*}{$P_{c}$} \\
\hline & & G0 & G1-2 & G3-5 & \\
\hline \multirow{3}{*}{ 焉 } & G0 & 12 & 0 & 0 & $100 \%$ \\
\hline & G1-2 & 0 & 11 & 1 & $91.7 \%$ \\
\hline & G3-5 & 0 & 7 & 14 & $66.7 \%$ \\
\hline
\end{tabular}

Figure 6: (a) and (b) Confusion matrices obtained from the separation in training and validation groups when considering the Young's modulus $E$ alone and the spectral-QUS parameters alone. (c), (d) and (e) Confusion matrices obtained from the leave-one-out cross-validation when considering the Young's modulus $E$ alone, the spectral-QUS parameters alone and the combination of spectral-QUS parameters with Young's modulus. Also given are the percentages of correctly classified lobes, denoted $P_{c}$. 